\title{
CA 125 in biological fluids
}

\author{
Alexandra Gireadă ${ }^{1}$, Irina Bălescu², Nicolae Bacalbașa ${ }^{3}$ \\ 1"Carol Davila" Central Military Emergency Hospital, Bucharest, Romania \\ 2"Ponderas" Hospital, Bucharest, Romania \\ 3"Carol Davila" University of Medicine and Pharmacy, Bucharest, Romania
}

\section{ABSTRACT}

CA 125 is one of the most popular tumor markers usually synthetized by normal and malignant cells of different origins. Increased levels can be found in both benign and malignant conditions in fluids with different origins such as pleural effusion, ascites, ovarian cysts fluids, amniotic fluid and even pancreatic cyst fluids. This is a literature review of the largest studies regarding various conditions in which increased levels of CA 125 can be found and their clinical significance.

Key words: CA 125, amniotic fluid, cyst fluid, ascites, pleural effusion

\section{INTRODUCTION}

Numerous studies have been realized in order to test the utility of CA 125 determination in many fluids of the organism: ascites, pleura, pericardium, amniotic fluid, cyst fluid, bronchoalveolar fluid, compared to that of serum determination. The research was based on the fact that CA 125 is produced by different types of cells in the body, either benign or malignant, most of them lying in tissues derived from the müllerian epithelia (1).

\section{CA 125 in the pleural fluid}

The etiological diagnosis of pleural effusions is difficult because they can be caused by many types of neoplasms. The highest frequency belongs to lung cancer (30\%), which is followed by breast cancer and lymphomas. The most commonly used method for the diagnosis is the cytopathologic examination of the pleural fluid. It has a sensitivity of $50 \%$. When the needle biopsy of the pleura is used, the diagnostic sensitivity becomes $80 \%$. The next and more invasive procedure which can be used is thoracoscopy. A lot of research is done in order to see if the determination of tumor markers can help the diagnosis (2).

The two major causes of pleural effusions are malignancy and infection. The most common infection associated with pleural effusion is tuberculosis. Both entities are associated with increased values of CA 125 in serum and pleural fluid. A study realized in Iran in 2004-2005 took into account twenty-seven
Corresponding author:

Nicolae Bacalbasa, MD

Dimitrie Racoviță Street, no. 2,

Bucharest, Romania

E-mail: nicolae_bacalbasa@yahoo.ro 
patients affected by tuberculosis and 23 patients diagnosed with malignant tumors. The mean CA 125 concentration measured in the pleural fluid of the patients of the two groups was $159.1 \pm 214$, and 2149.2 $\pm 4513.6 \mathrm{U} / \mathrm{ml}$, respectively. The difference was statistically significant $(p<0.01)$, and thus CA 125 measured in pleural fluid can be used for the differential diagnostic of the two entities, both characterized by chronic pleural accumulation of fluid. The cut-off used for the differential diagnosis was determined by means of a ROC curve and it was $221.5-253.5 \mathrm{U} / \mathrm{ml}$ (3).

A study realized in Turkey in 2007 included 35 patients with malignant pleural effusions, 54 patients with benign pleural effusions and 25 healthy individuals in order to investigate the possible role of the serum and pleural fluid concentrations of CEA, CA 15-3, CA 19-9 and CA 125 in the diagnosis. The concentrations were statistically higher in patients with malignant diseases than in patients with benign affections and patients from the control group. For the detection of malignancy, CEA had the highest sensitivity, specificity and accuracy: $41.6 \%, 100 \%$ and $74.1 \%$, respectively. The values of CA $15-3$ are similar to those of CEA: $38.8 \%, 100 \%$ and $73 \%$, respectively. CA 19-9 and CA 125 had lower values. The association of CEA, CA $15-3$ and CA 125 had a specificity of $100 \%$ and an accuracy of $78.3 \%$. The association of CA 15-3 and CA 125 had the highest diagnostic accuracy: $80.9 \%$, with a specificity of $100 \%$. It is known from the literature that the sensitivity of the cytologic exam is about $50-70 \%$ and the accuracy of thoracoscopy is about $90 \%$, the last being an invasive an expensive investigation. The results showed that the patients diagnosed with lung cancer had higher pleural levels of CEA and CA 15-3 than patients diagnosed with extrapulmonary cancer. An idea suggested by the authors is that the increased concentration of CEA in pleural fluid in malignant conditions can be caused by the obstruction of the lymphatics and by pleural invasion. In conclusion, the authors approved the use of the combinations of tumor markers for the diagnose of malignant pleural fluids (4).

The use of other tumor markers is also investigated. A study realized in 2007 in Brazil determined the concentrations of the tumor markers CEA (carcinoembryonic antigen), CYFRA21-1 (cytokeratin 19 fragment) and CA 15-3 (carbohydrate antigen) in the blood and pleural fluid in order to estimate their role in the diagnosis of pleural effusions. It included 36 patients with malignant effusions, 30 patients with benign diseases and 19 patients with inconclusive results. In patients with malignancies, CEA and CYFRA21-1 had higher values in the pleural fluid than in the blood. In patients with benign diseases, CYFRA21-1 had higher values in the pleural fluid than in the blood; CEA and CA 15-3 had higher values in the blood than in the pleural fluid. The diagnostic sensitivities for all these markers, at a specificity of $>90 \%$, in the pleural fluid, were 69.4 for CEA, 69.4 for CYFRA21-1, 66.7 for CA 153 and $80.6 \%$, the combined sensitivity. In blood, the corresponding values were 57.1 for CEA, 71.4 for CYFRA21-1, 48.6 for CA $15-3$ and $77 \%$ the combined sensitivity. The authors concluded that the markers can be used for the diagnosis in patients with pleural effusions (2).

The production of CA 125 by the pleural cells is another research theme. It is sustained by the presence of increased serum and pleural fluid CA 125 concentrations in patients diagnosed with various diseases affecting pleura. There are cases described in the literature in which an elevation of serum and pleural fluid CA 125 can be associated with sarcoidosis. One of these is of a 55-year-old woman, who had a concentration of $2,846.8 \mathrm{U} / \mathrm{mL}$ in the pleural fluid and $229.5 \mathrm{U} / \mathrm{ml}$ in the serum. These values turned back to normal after steroid therapy.In this case, a possible differential diagnosis can be done with tuberculosis, in which massive pleural effusions can be associated with increased CA 125 serum concentrations(5).

\section{CA 125 in the ascitic fluid}

As in the case of pleural fluid, numerous studies investigate the role of CA 125 in the differential diagnosis of various conditions associated with ascites.

A study realized in 2009 in Turkey took into account 55 patients with malign ascites and 36 patients with benign ascites. It measured the concentration of several tumor markers in the serum and in the ascitic fluid: CEA (carcinoembryonic antigen), CA (cancer antigen) 125, CA 19.9, CA 72.4, CA 15.3, AFP ( $\alpha$-fetoprotein) and CYFRA (cytokeratin-19 fragment). The patients presenting with malignancies had statistically significant differences between the serum and the ascitic fluid concentrations of CEA, CA 125, CYFRA and AFP, whilst the patients presenting with benign conditions had statistically significant differences between the serum and the ascitic fluid concentrations of CEA, CA 19.9, CA 15.3, CYFRA and AFP. The idea of the study came from the fact that the sensitivity of cytology in the etiologic diagnosis of ascites is of only $50 \%$. The samples were analyzed using an electrochemiluminescence immunoassay and the cytological examination of ascitic fluid was realized by means of haematoxylin and eosin and of Giemsa stains. There were also statistically significant differences between the benign and malign group 
regarding serum and ascitic fluid concentrations of the following markers: CEA, CA 19.9,CA 72.4, CYFRA and AFP. The results showed that, with the exception of CYFRA, the serum and the ascitic fluid levels of the tumor markers in patients with malignant affections were well correlated, leading to the conclusion that the measurement of ascitic fluid tumor markers in patients with malign conditions is not necessary. Another argument which supports this idea is the production of CA 125 , CYFRA and CEA by the normal mesothelial cells. Of much utility is also the serum AFP concentration, which was higher than that of the ascitic fluid and which can detect hepatocellular carcinoma with a higher sensitivity than the ascitic cytology (6).

A study realized between 2000 and 2002 in Iran included 33 patients with cirrhosis, 6 patients diagnosed with malignancy, 5 patients with nephritic syndrome, 9 patients with tuberculosis peritonitis and 29 control patients. Their serum and ascitic fluid CA 125 concentrations were measured, as an alternative to more expensive investigations like laparoscopy, histology and tissue culture. The results showed a normalization of the serum CA 125 level in patients with tuberculosis peritonitis after the anti-tubercular treatment. They showed also increased serum and ascitic fluid CA 125 levels in the majority of the patients with cirrhosis. All the patients with malignancy had elevated serum CA 125 concentrations and about two thirds of them had elevated ascitic fluid levels. These results can sustain the possibility that the peritoneum is a source of CA 125 production. The control patients and those with nephrotic syndrome had normal serum and ascitic fluid CA 125 levels. These results can be explained by the urinary excretion of CA 125 in nephrotic syndrome (7).

A study realized by M.Epiney et al in 2000 has investigated in vivo and in vitro the production of CA 125 by the peritoneum and its contribution to increased serum CA 125 levels. Its results showed a raise in serum CA 125 concentrations after a peritoneal surgery and demonstrated that this raise was greater than in patients who undergone a non-peritoneal surgery. The results proved the in-vivo production of CA 125 by the peritoneum (8).

As a consequence of this existing research, Sevinc $A$ et al concluded that a careful interpretation may be necessary in patients presenting with increased serum CA 125 concentrations and serosal involvement, but without a tumor mass (9).

The ideas exposed above are confirmed by increased CA 125 levels in various conditions affecting the peritoneum.
In an article published in 2013 by R.B. Khalil et al, the authors describe a case of severe hypothyroidism having refractory ascites and increased serum CA 125 value $(1255 \mathrm{U} / \mathrm{mL})$ as a unique manifestation. Given the fact that CA 125 values in such situations can be as high as those encountered in malignancies, they suggest that the thyroid function should be evaluated in patients with ascites and high serum CA 125 levels, in order to avoid the use of diuretics, paracentesis, liver biopsies and exploratory laparotomies. There are two possible explanations for the increased serum levels of CA 125: the stretching of the peritoneum with ascites and the lymphatic absorption of ascites, which brings into the circulation the CA-125 antigen associated with the peritoneal cells. The normalization of CA 125 concentrations and the complete regression of the ascites can be achieved by the hormone replacement therapy (10).

\section{CA 125 levels in the ascitic fluid as compared to pleural effusions}

A study realized in India in 2006 determined the CA 125 concentration in 38 pleural samples and 46 ascitic fluid samples from patients suffering from non-malignant conditions and in 10 serum samples from patients with pulmonary tuberculosis in order to evaluate the non-specific rise of this marker and to explain the mechanism of its secretion. The ELISA method was used and the results showed increased CA 125 concentrations in both transudative and exudative ascitic and pleural fluid samples, with significantly higher CA 125 levels in the ascitic fluid, which proves the greater capacity of the peritoneum to secrete CA 125 as a consequence of inflammation or of mechanical distress (11).

The idea is sustained by the research effectuated by Mollina et al, who considered the presence of ascites as a possible explanation for the raised serum CA 125 concentrations, given that the CA 125 levels in the ascitic fluid were higher than those in the serum (the case of bacterial peritonitis)(12).

In the present study, important data were obtained in patients having pulmonary tuberculosis without the involvement of pleura. In their cases, pleural CA 125 concentrations were not increased. Kalantri et al included in a study patients with tuberculous or pyogenic serosal infections. The results showed that all the patients with ascites and 70 per cent of the patients with pleural effusions had increased body fluid levels of CA 125 . The levels were higher in ascitic fluid as compared to the pleural effusion and these results prove the higher capacity of the peritoneal epithelium to secrete CA 125 as 
compared to the pleural epithelium (11).

In an article published in 2007, S.F. Hussain \& P. Camilleri wrote that, from an oncologist point of view, determining the CA 125 concentration in the pleural and ascitic fluid doesn't help the differential diagnosis between different malignant tumours or between benign and malignant diseases. They concluded also that, from a researcher's point of view, besides the role of CA 125 in the ovarian cancer, there may be also a lot to know about its production by different tissues of the body and other possible utilities (13).

\section{CA 125 in body fluids involved in endometriosis}

The capacity of the endometrium to produce and secrete CA 125 can explain the increased serum CA 125 concentrations in patients with endometriosis. Although these concentrations rarely exceed 100 $\mathrm{IU} / \mathrm{ml}$, the levels encountered within the cystic fluid can be higher than $1000000 \mathrm{U} / \mathrm{ml}$ and this is due to the protection of the cyst by a thick wall which prevents the content to enter the circulation. However, higher serum values are found when this wall is affected (ruptured endometriomas) and in unruptured endometriomas, but which are in an advanced stage, which have an enlarged surface area of endometrial tissue, are associated with endometrial foci on the omentum or are characterized by adhesions in the peritoneum (14-16).

A study published in 1992 investigated the role of CA 125 "chocolate" cyst fluid measurement in the differential diagnosis of the endometriotic cyst and the cystic corpus luteum. The study included 53 women with cysts. The liquid was aspirated by means of an echo-guided puncture for in-vitro fertilization and the concentration of CA 125 and of other parameters was measured in the fluids with a dark-brown appearance. These 42 patients also undergone a cystectomy and the diagnosis were of endometrioma in $68 \%$ of the cases, of corpora lutea in $27 \%$ of the cases and of follicular cysts in $5 \%$ of the cases. $78 \%$ of the CA 125 concentrations in the endometriotic cysts were very high (>10,000 IU/ml), the rest of them were lower than $1000 \mathrm{IU} / \mathrm{ml}$, as they were in the liquids from corpora lutea. In contrast to the endometriotic cysts, these latest cysts were characterized by increased 17 beta-oestradiol levels (> $2000 \mathrm{pg} / \mathrm{ml}$ ) and/or increased progesterone levels (> $100 \mathrm{ng} / \mathrm{ml}$ ). The authors concluded that the determination of CA 125 when a dark brown fluid is aspirated during the echo-guided puncture can be useful in establishing the diagnosis(16).

\section{CA 125 in the ovarian cyst fluid}

The determination of CA 125 in the ovarian cyst fluid may have a possible role in the assessment of prognosis in patients with ovarian adenocarcinoma. A study realized in Netherlands in 2009 included 54 patients and determined the CA 125 levels in cyst fluid and serum. The median concentrations obtained were of $55,500 \mathrm{U} / \mathrm{ml}$ (with a range of 590-10,200,000 U/ml) and $179 \mathrm{U} / \mathrm{ml}$ (with a range of $13-11,000 \mathrm{U} / \mathrm{ml}$ ) respectively. The cyst fluid concentrations were always higher than those of serum $(p<0.001)$ and between the two groups of values there was a moderate correlation $(R=0.337, p=0.016)$. The CA 125 levels in the ovarian cyst fluid were correlated with tumor grade $(p=0.038)$, with histology $(p<0.001)$ and the levels above the median were well correlated with a poor disease-free survival. This later parameter was associated with FIGO stage, tumor grade $(p=0.025)$, serous histology $(p=$ $0.003)$ and increased serum CA $125(p=0.009)$ in univariate analysis. However, the conclusion was that the cyst fluid CA 125 cannot be used as a prognostic biomarker because only FIGO stage proved to have an independent predictive value in multivariate analysis. The study showed that the concentration of ovarian cyst fluid CA 125 in patients with mucinous tumors was significantly lower than in patients presenting with other histological types $(p<0.001)$ and the CA 125 cyst fluid levels in patients with well-differentiated tumors was significantly lower than in patients presenting with poorly-differentiated tumors $(p=0.038)(17)$.

\section{CA 125 in the pancreatic cyst fluid}

Another utility of CA 125 determination is the differential diagnosis of the pancreatic cysts, which can be inflammatory pseudocysts (most of them), cysts of tumoral nature (serous and mucinous) and various rare cystic lesions. The determination of the concentrations of CEA, CA 125 and amylase in the cyst fluid, together with the determination of the viscosity, have an important diagnostic role, although more research is still necessary(18).

A study published in 1992 by M.R. Pins et al presen-ted the case of an enteric duplication cyst, having a probable foregut origin. Its differential diagnosis with a pancreatic cyst of malignant origin was difficult to make because of the increased concentrations of CEA, CA 125, amylase and of the increased viscosity of the cyst fluid. Although elevated amylase levels are a characteristic of the pseudocysts, they can also be found in cysts of malignant origin. This article is an argument for the possible role of CA 125 in the 
diagnosis of non-malignant rare pancreatic cystic lesions (18).

\section{CA 125 in the amniotic fluid}

The development of the proteomics technology has led to the discovery of many biomarkers which can be determined in the maternal serum for a possible diagnosis of the catastrophic syndrome named amniotic fluid embolism. Between them there are some tumor markers like CA 125, (interleukin) IL-6, SCC (squamous cell carcinoma), insulin-like growth factor-binding protein (IGFBP)-1 and osteopontin. In the amniotic fluid samples, the mean CA 125 concentration is of 700 $\mathrm{U} / \mathrm{ml}$, the mean concentration in the maternal serum being $6 \mathrm{U} / \mathrm{ml}(\mathrm{AF} / \mathrm{MS}$ ratio $=100)$. A study showed that $10 \%$ of the CA 125 serum concentrations in pregnant women were above cut-off $(>35 \mathrm{U} / \mathrm{ml})$. Further research is still necessary for establishing the diagnostic value of these markers (19).

The determination of CA 125 from the amniotic fluid may have other possible roles. A study published in June 2015 took into account 36 women who were supposed to have an amniotic fluid infection and who were expected to have a preterm delivery. The authors measured the amniotic fluid concentrations of several parameters: MMP-8 (matrix metalloproteinase-8), WBC (white blood cells), glucose, CA 125 and performed an amniotic fluid culture and PCR. The results showed that CA 125 levels were of about $5608 \pm$ $864 \mathrm{IU} / \mathrm{ml}$ (mean \pm standard deviation) in patients with IAI (intra-amniotic inflammation) and of $904 \pm 84 \mathrm{IU} / \mathrm{ml}$ in non-IAI patients. $(p=0.001)$. They also showed a positive correlation between the CA 125 levels and the WBC and the MMP-8 levels in the amniotic fluid and a negative correlation between the CA 125 levels and the gestational age. Patients diagnosed with MIAC (microbial invasion of the amniotic cavity) had higher levels of CA 125 in the amniotic fluid $(p=0.064)$. The authors found that for a cut-off of $1650 \mathrm{IU} / \mathrm{ml}, \mathrm{CA} 125$ has a sensitivity of $71.4 \%$ and a specificity of $86.4 \%$ for the prediction of delivery within one week after the measurements. They concluded that the raised CA 125 concentrations in the amniotic fluid can be a sign of an imminent preterm delivery, since they can be found in patients with amniotic fluid inflammation (20).

\section{CA 125 in the follicular fluid}

Research was also done in order to investigate thequality of the oocyte used for the human in vitro fertilization procedure. A study realized in 2008 measured the concentrations of several hormones, bone morphogenetic protein-15, insulin-like growth factors, interleukins, reactive oxygen species, growth factors, anti-apoptotic factors, proteinic components, other molecular factors and tumor markers in the follicular fluid. The results of the study showed that the concentrations of AFP, CEA and CA 125 in the follicular fluid are not related to the oocyte quality $(21,22)$. Another study proved that these concentrations don't influence the outcome of the IVF cycle(22).

\section{CA 125 variability depending on the producing tissues}

A study realized in Serbia in 2007 tried to describe the antigenic structure of CA 125 using various methods: gel filtration, ion-exchange chromatography, peptide mass fingerprinting and lectin-binding assays. It get to the conclusion that the molecule produced by the amniotic epithelium has a very heterogeneous structure, showing subtle differences from the CA 125 produced in cancer and from CA 125 produced by the placental tissue (23).

These results have also been shown by other studies, which proved that different tissues have different ways of production and/or metabolism of CA 125 and all the studies mentioned above can sustain the idea that all the research done on this theme is justified by the clinical utility of CA 125 determination from various parts of the body (24).

\section{CONCLUSIONS}

The presence of increased level of CA 125 in biological fluids is a common situation for both malignant and benign conditions. Most often increased levels of CA 125 can orientate the diagnosis to certain pathology but sometimes other specific biological tests related to the patients' clinical symptoms should be associated in order to establish the right diagnosis.

\section{REFERENCES}

1. Molina R, Filella X, Jo J, Agusti C, Ballesta AM. CA 125 in biological fluids. Int J Biol Markers 1998 Oct; 13(4):224-30.

2. Wagner IC, Guimaraes MJ, da Silva LK, de Melo FM, Muniz MT. Evaluation of serum and pleural levels of the tumor markers CEA, CYFRA21-1 and CA 15-3 in patients with pleural effusion. J Bras Pneumol 2007 Mar; 33(2):185-91.

3. Shokouhi S, Abadi MS, Gachkar L. Pleural Fluid CA-125 in Patients with Pleural Effusion, Tanaffos 2005; 4(16):23-7.

4. Pasaoglu G, Zamani A, Can G, Imecik 0. Diagnostic value of CEA, CA-199, CA 125 and CA 15-3 levels in malignant pleural fluids. Eur J Gen Med 2007; 4(4):165-71

5. Lee IS, Kim SB, Moon CS, Jung SM, Kim SY, Kim EY, Jung JY, Kang YA, Kim YS, Kim SK, Chang J, Park MS. Sarcoidosis presenting with massive pleural effusion and elevated serum and pleural fluid carbohydrate antigen125 levels. TubercRespir Dis (Seoul ) 2012 Dec; 73(6):320-4.

6. Tuzun Y, Celik Y, Bayan K, Yilmaz S, Dursun M, Canoruc F. Correlation of tumour markers in ascitic fluid and serum: are measurements of ascitic- 
tumour markers a futile attempt? J Int Med Res 2009 Jan; 37(1):79-86.

7. Azmi M, Ghanavati M, Keramati MR, Bashashati M, Hajiani E, Masjedi A, Hashemi SJ. Serum and ascitic fluid CA-125 level in patients with ascites. Indian J Gastroenterol 2007 Jan; 26(1):45-6.

8. Epiney M, Bertossa C, Weil A, Campana A, Bischof P. CA125 production by the peritoneum: in-vitro and in-vivo studies. Hum Reprod 2000 Jun 15(6):1261-5

9. Sevinc A, Camci C, Turk HM, Buyukberber S. How to interpret serum CA 125 levels in patients with serosal involvement? A clinical dilemma. Oncology 2003; 65(1):1-6.

10. Bou KR, El Rassi P, Chammas N, Obeid J, Ghabach M, Sakr C, Itani T, Farhat S. Myxedema ascites with high CA-125: Case and a review of literature. World J Hepatol 2013 Feb; 5(2):86-9.

11. Kalantri $Y$, Naik G, Joshi SP, Jain A, Phatak S, Chavan R, Hemvani N, Chitnis DS. Role of cancer antigen-125 from pleural \&ascitic fluid samples in non malignant conditions. Indian J Med Res $2007 \mathrm{Jan} ; 125(1): 25-30$

12. Molina R, Filella X, Bruix J, Mengual P, Bosch J, Calvet X, Jo J, Ballesta AM Cancer antigen 125 in serum and ascitic fluid of patients with liver diseases. ClinChem 1991 Aug; 37(8):1379-83.

13. Hussain SF, Camilleri P. Elevation of tumour marker CA-125 in serum \& body fluids: interpret with caution. Indian J Med Res 2007 Jan; 125(1):102.

14. Hosseini MA, Aleyasin A, Khodaverdi S, Mahdavi A, Najmi Z. Extra-ordinary High CA-125 and CA 19-9 Serum Levels in an Ovarian Endometrioma: Case Report. Journal of Family and Reproductive Health 2009 Jun; 3(2).

15. Johansson J, Santala M, Kauppila A. Explosive rise of serum CA 125 following the rupture of ovarian endometrioma. Hum Reprod 1998 Dec; 13(12):3503-4.

16. Koninckx PR, Muyldermans M, Moerman P, Meuleman C, Deprest J,
Cornillie F. CA 125 concentrations in ovarian 'chocolate' cyst fluid can differentiate an endometriotic cyst from a cystic corpus luteum. Hum Reprod 1992 Oct; 7(9):1314-7.

17. Kolwijck E, Span PN, Thomas CM, Bulten J, Sweep FC, Massuger LF. Prognostic value of CA 125 in ovarian cyst fluid of patients with epithelial ovarian cancer. Oncol Rep 2010 Feb; 23(2):579-84.

18. Pins MR, Compton CC, Southern JF, Rattner DW, Lewandrowski KB. Ciliated enteric duplication cyst presenting as a pancreatic cystic neoplasm: report of a case with cyst fluid analysis. ClinChem 1992 Aug; 38(8 Pt 1):1501-3.

19. Kobayashi H, Naruse K, Sado T, Noguchi T, Yoshida S, Shigetomi H et al. Search for Amniotic Fluid-Specific Markers: Novel Biomarker Candidatesfor Amniotic Fluid Embolism. The Open Women's Health Journal, 2011; 5:715.

20. Seong WJ. Amniotic fluid CA-125 as a marker of intra-amniotic inflammation associated with preterm delivery: a preliminary single center study. Arch GynecolObstet 2015 Jun.

21. Revelli A, Delle PL, Casano S, Molinari E, Massobrio M, Rinaudo P. Follicular fluid content and oocyte quality: from single biochemical markers to metabolomics. ReprodBiolEndocrinol 2009; 7:40.

22. Mordel N, Anteby SO, Zajicek G, Roisman I, Treves A, Barak V. CA-125 is present in significant concentrations in periovulatory follicles of in vitro fertilization patients. FertilSteril 1992 Feb; 57(2):377-80.

23. Milutinovic B, Jankovic M. Analysis of the protein and glycan parts of CA125 antigen from human amniotic fluid, Arch BiolSci, Belgrade 2007; 59 (2):97-103.

24. Barbati A, Lauro V, Orlacchio A, Cosmi EV. Immunoblotting characterization of CA 125 in biological fluids: difference between pregnancy and cancer CA 125 origin. Anticancer Res 1996 Nov; 16(6B):3621-4. 